\title{
Sequential treatment from mandibulectomy to reconstruction on mandibular oral cancer - Case review II: mandibular anterior and the floor of the mouth lesion of basaloid squamous cell carcinoma and clear cell odontogenic carcinoma
}

\author{
Jae-Young Yang, Dae-Seok Hwang, Uk-Kyu Kim \\ Department of Oral and Maxillofacial Surgery, School of Dentistry, Pusan National University, Yangsan, Korea
}

\begin{abstract}
J Korean Assoc Oral Maxillofac Surg 2021;47:216-223)
Preoperative patient analysis for oral cancer involves multiple considerations that are based on multiple factors; these include TNM stages, histopathologic findings, and adjacent anatomical structures. Once the decision is made to excise the lesion, the margin of dissection and its extent should be considered along with the best form of reconstruction and airway management. Treatment methods include surgical resection, radiotherapy, and chemotherapy. Although the combined method of treatment is controversial, surgical resection is considered predominantly, and immediate reconstruction after surgical resection follows. The choice of treatment is dictated by the anticipated functional and esthetic results of treatment and also by the availability of a surgeon with the required expertise. Segmental mandibulectomy with primary reconstruction has been shown to have advantages in both functional and esthetic results. A 52-year-old male patient with basaloid squamous cell carcinoma of the floor of the mouth, and the anterior portion of the mandible was treated with surgical procedures that included segmental mandibulectomy with both supraomohyoid neck dissection (SOHND) at Levels I-III and mandible reconstruction with a left fibula free flap. A 55-year-old male patient with clear cell odontogenic carcinoma of the oral cavity underwent segmental mandibulectomy with both SOHND at Levels I-III and mandible reconstruction with a left fibula free flap. The purpose of this study was to review the anatomic and functional results of patients after immediate reconstruction with a fibula free flap following resection of carcinoma in the anterior portion of the mandible and floor of the mouth.
\end{abstract}

Key words: Segmental mandibulectomy, Free tissue flap, Carcinoma, Fibula

[paper submitted 2020. 9. 29 / revised 2020. 12. 7 / accepted 2020. 12. 11]

\section{Introduction}

Oral cancer accounts for approximately $6 \%$ of all cancers; this percentage varies depending on race, geographic area, and sex. Oral cancer can affect all areas of the mouth, including the tongue, lip, alveolar ridge, buccal mucosa, and floor of the mouth. Prognosis varies based on anatomical location. Among all oral cavity cancers, advanced cancer of the tongue

\footnotetext{
Uk-Kyu Kim

Department of Oral and Maxillofacial Surgery, Pusan National University Dental Hospital, School of Dentistry, Pusan National University, 20 Geumoro, Mulgeum-eup, Yangsan 50612, Korea

TEL: +82-55-360-5100

E-mail:kuksjs@pusan.ac.kr

ORCID: https://orcid.org/0000-0003-1251-7843

(c) This is an open-access article distributed under the terms of the Creative Commons Attribution Non-Commercial License (http://creativecommons.org/ licenses/by-nc/4.0/), which permits unrestricted non-commercial use, distribution, and reproduction in any medium, provided the original work is properly cited.

Copyright (C) 2021 The Korean Association of Oral and Maxillofacial Surgeons. All rights reserved.
}

and floor of the mouth is associated with poor prognosis and a high risk of recurrence due to involvement of several vital structures at these anatomical sites ${ }^{1,2}$.

Treatment of oral cancer includes surgical resection, radiotherapy, and chemotherapy. Although the role of combined therapy remains controversial, surgical resection should be considered as the predominant therapeutic option, and immediate post-resection reconstruction is essential.

Mandibular reconstruction is a complicated and challenging procedure in the field of reconstructive craniomaxillofacial surgery. The ultimate goal of this surgery is structural and functional restoration of the mandible, which necessitates simultaneous evaluation of appearance, mastication, speech, and oral function. The surgeon can achieve these ends with a variety of surgical techniques, mainly through the use of vascularized grafts.

In this study, we describe cancer of the floor of the mouth and the anterior mandible in two patients who underwent seg- 
mental treatment, including mandibulectomy and reconstruction with a fibula free flap, for mandibular basaloid squamous cell carcinoma (BSCC) and clear cell odontogenic carcinoma (CCOC), respectively. We also present a literature review.

\section{Case Presentation}

\section{Case 1}

A 52-year-old man visited the Department of Oral and Maxillofacial Surgery at Pusan National University Dental Hospital (Yangsan, Korea) in March 2018, with pain in the lower incisors and the floor of the mouth. Physical examination showed a red, proliferative, ulcerated lesion on the floor of the mouth and anterior portion of the mandible, and palpation revealed right-sided submandibular lymphadenopathy.(Fig. 1. A) Histopathological evaluation of a biopsy performed at a previous hospital showed BSCC or adenoid cystic carcinoma. We performed a repeat biopsy, which confirmed the diagnosis of BSCC.(Fig. 2) The patient's medical history was remarkable only for heavy smoking. Routine laboratory test results were unremarkable, and positron emis- sion tomography (PET), computed tomography (CT), and magnetic resonance imaging (MRI) were performed. We observed a red, proliferative, ulcerated lesion $(4-6 \mathrm{~cm})$ on the floor of the mouth; this lesion extended to the lower anterior gingiva. Orthopantomography revealed an ill-defined lesion of the anterior mandible.(Fig. 1. B) CT and facial MRI showed an enhancing density around the left anterior mandible that extended to both the sublingual space and the left tongue base, as well as bony erosions of the symphysis and the left-sided alveolar process of the mandible.(Fig. 1. C). Fluorodeoxyglucose PET-CT indicated an abnormally high uptake in the floor of the mouth (maximum standardized uptake value [SUVmax]: 17.1) and in both Level IB lymph nodes (LNs) (SUVmax: 11.9 on the right and 3.2 on the left). (Fig. 1. D)

We performed successful segmental mandibulectomy with supraomohyoid neck dissection (SOHND) along with Levels I-III and mandibular reconstruction using a left fibula free flap under general anesthesia, followed by radiotherapy.(Fig. 3) Postoperatively, the patient received six weeks of radiotherapy (50 Gy fractions) to treat regional LN metastasis. He has shown a good prognosis and is being followed.(Fig. 4)
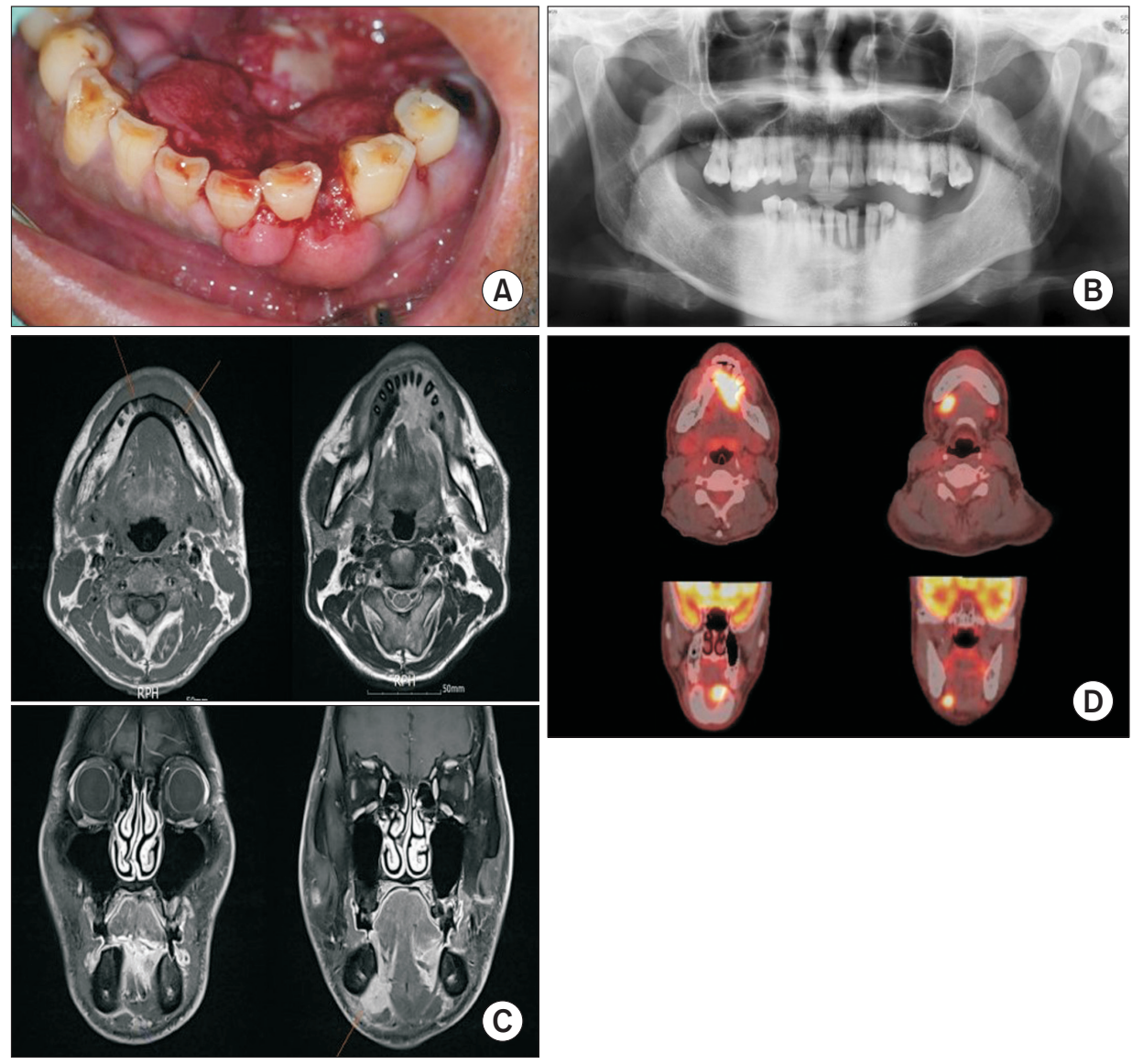

Fig. 1. Preoperative clinical and radiographic features. A. Intraoral feature. Ulcerative and proliferative lesion on the floor of mouth and the anterior of mandible. B. Panoramic radiograph image. Ill-defined osteolytic lesion on mandibular anterior. C. Facial magnetic resonance image showed enhancing soft tissue density around the mandibular anterior, extend to both sublingual space and left tongue base and bony erosive change of mandibular symphysis. Multiple enhancing bilateral lymph nodes (LNs) (Level IA, IB) were observed. D. Abnormally increased fluorodeoxyglucose uptake was observed in the floor of mouth (standardized uptake value [SUV]: 17.1) and in both Level IB LNs (SUV: 11.9 and 3.2, respectively). Jae-Young Yang et al: Sequential treatment from mandibulectomy to reconstruction on mandibular oral cancer - Case review II: mandibular anterior and the floor of the mouth lesion of basaloid squamous cell carcinoma and clear cell odontogenic carcinoma. $J$ Korean Assoc Oral Maxillofac Surg 2021 

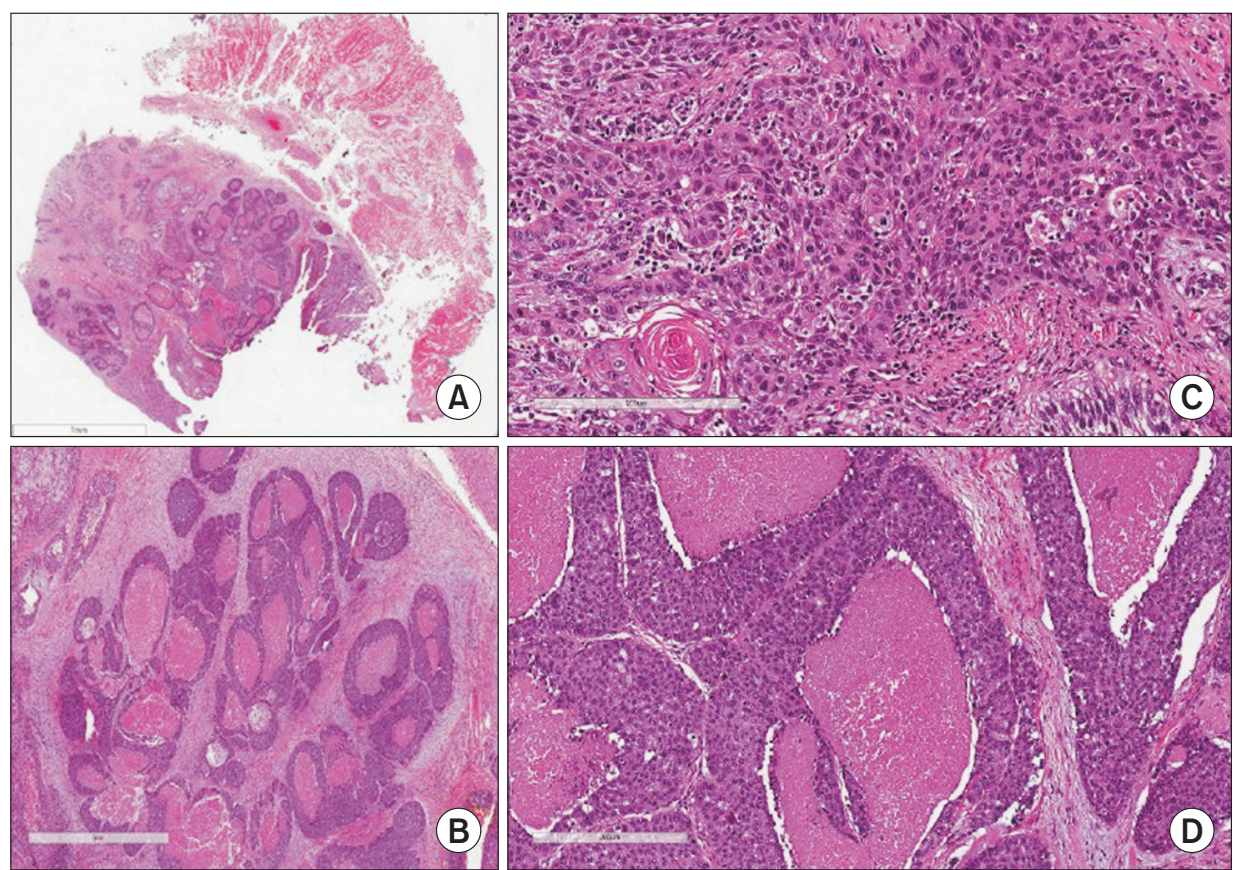

Fig. 2. Representative histological section of basaloid squamous cell carcinoma. H\&E staining, $A: \times 0.4$ (scale bar=7 mm), B: $\times 2$ (scale

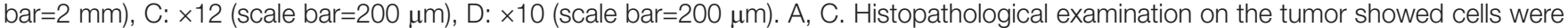
forming nodule and growing. There were discrete cell boundaries, abundant eosinophilic cytoplasm, and a large number of tumor cells in which the nucleus was very distinct and the nucleoli were well visible. Nuclear hyperchromatism and pleomorphism were evident and many mitoses were observed. Dysplastic epithelial cells with keratin pearl formation are clearly evident and are considered to be well differentiated squamous cell carcinoma. B, D. Cell nest or lobule, forming infiltrative growth. Characteristically comedo-type necrosis was observed.

Jae-Young Yang et al: Sequential treatment from mandibulectomy to reconstruction on mandibular oral cancer-Case review II: mandibular anterior and the floor of the mouth lesion of basaloid squamous cell carcinoma and clear cell odontogenic carcinoma. J Korean Assoc Oral Maxillofac Surg 2021
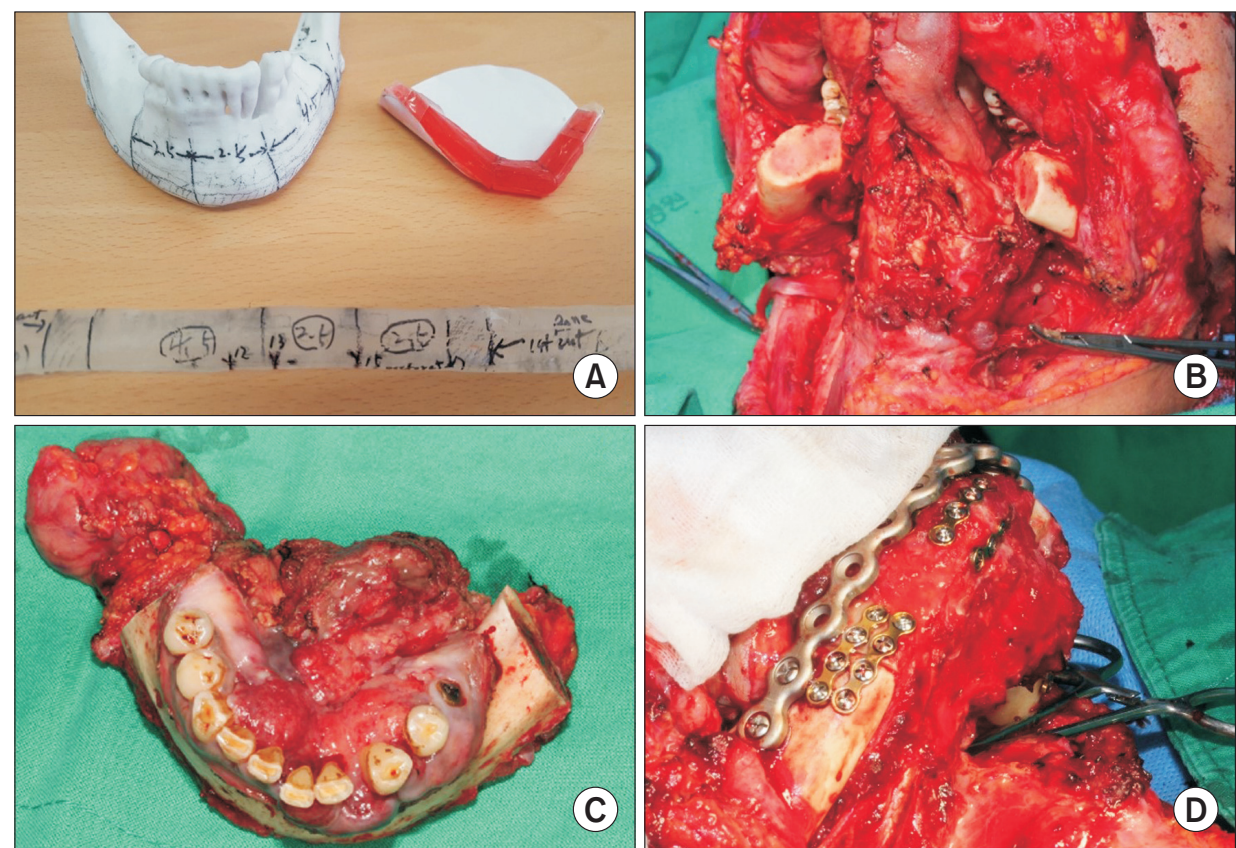

(C)

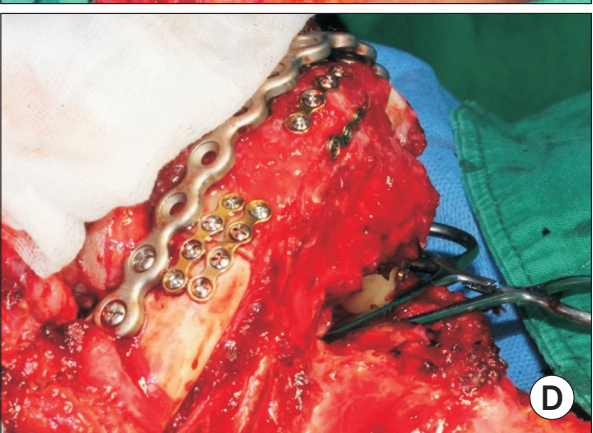

Fig. 3. Intraoperative photographs. A. Rapid prototype model of mandible, resin block for fibula osteotomy. B. Segmental mandibulectomy with supraomohyoid neck dissection. C. Excised tumor mass including mandible, the floor of the mouth, lymph node. D. Mandibular reconstruction with fibula free flap fixed with reconstruct plate and mini plates.

Jae-Young Yang et al: Sequential treatment from mandibulectomy to reconstruction on mandibular oral cancer - Case review II: mandibular anterior and the floor of the mouth lesion of basaloid squamous cell carcinoma and clear cell odontogenic carcinoma. J Korean Assoc Oral Maxillofac Surg 2021 


\section{Case 2}

A 55-year-old man was admitted to the Department of Oral and Maxillofacial Surgery at Pusan National University Dental Hospital in September 2016, with pain in the anterior portion of the mandible. His medical history was remarkable only for hypertension. Laboratory test results were unremarkable.

Orthopantomography and cone-beam CT revealed an irregular erosive lesion $(38 \mathrm{~mm} \times 31 \mathrm{~mm})$ in the anterior portion of the mandible.(Fig. 5) Based on histopathological examination of a biopsy specimen obtained under local anesthesia, the lesion was diagnosed as ameloblastoma by the Department of Pathology of Pusan National University Yangsan Hospital. (Fig. 6)

Wide excision and mandibular reconstruction with iliac block bone graft were performed under general anesthesia to avoid the risk of recurrent ameloblastoma.(Fig. 7) Based on detection of multiple clear cells upon histopathological evaluation of the resected mass, as well as the clinical features, the lesion was diagnosed by the Department of Oral Pathology of the School of Dentistry at Pusan National University as CCOC instead of ameloblastoma.(Fig. 8) CT, MRI, and PET-CT were performed to confirm an accurate evaluation one month postoperatively.(Fig. 9) We performed successful SOHND along with Levels I-III and mandibular reconstruction with a left fibula free flap under general anesthesia.(Fig. 10) Histopathological evaluation of an intraoperative frozen biopsy section demonstrated normal tissue findings. The permanent biopsy results showed negative findings in the marginal and neck areas with potential metastasis, excluding the resection site. The surgical site healed satisfactorily, and no adjuvant chemotherapy or radiation therapy was required. The patient has been regularly followed-up and has shown no recurrence to date.(Fig. 11)

\section{Discussion}

In this study, we retrospectively investigated two patients with oral cancer (one with cancer of the floor of the mouth and the other with anterior mandibular cancer) who were surgically treated at Pusan National University Dental Hospital. Based on histopathological evaluation, the patients described in Cases 1 and 2 were diagnosed with BSCC and CCOC, respectively.

BSCC is a common malignancy that primarily affects the skin in humans and accounts for approximately $75 \%$ to $80 \%$
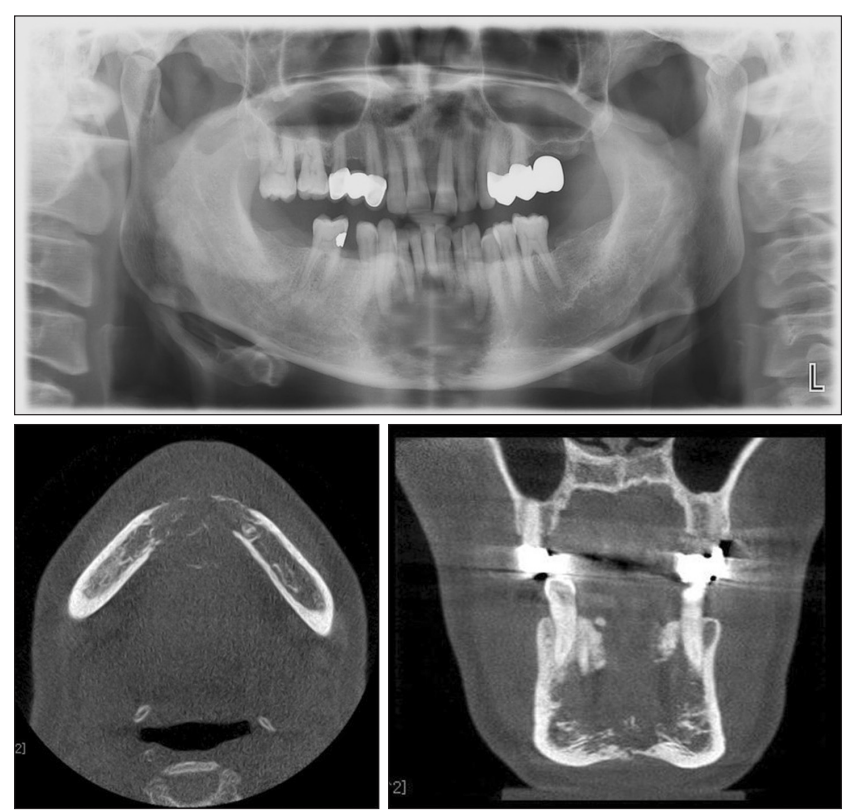

Fig. 5. Preoperative radiographs of panorama \& cone-beam computed tomography. In the anterior portion of the mandible, irregularly lesions with a diameter of $3 \mathrm{~cm}$ are observed. Perforation of the bucco-lingual cortical bone and root resorption are observed. Jae-Young Yang et al: Sequential treatment from mandibulectomy to reconstruction on mandibular oral cancer -Case review II: mandibular anterior and the floor of the mouth lesion of basaloid squamous cell carcinoma and clear cell odontogenic carcinoma. J Korean Assoc Oral Maxillofac Surg 2021
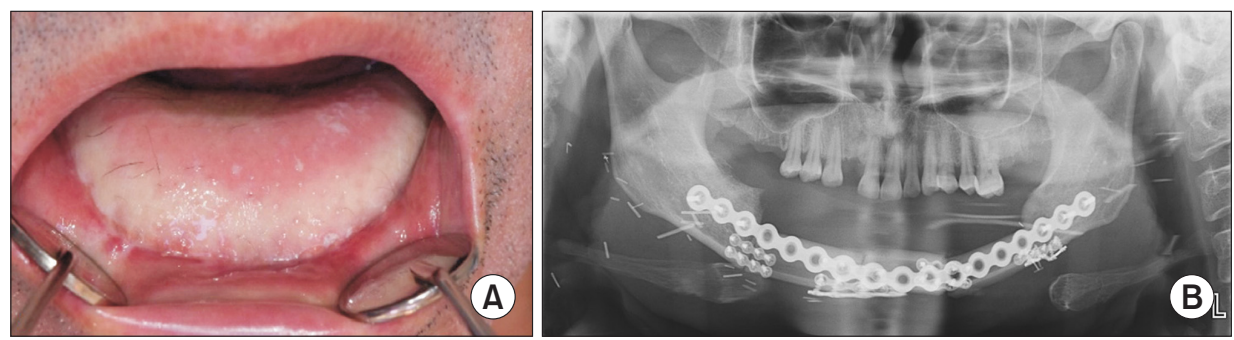

Fig. 4. Postoperative findings. A. Intraoral photograph of the primary site. B. Panoramic radiography. The patient underwent radiotherapy over 6 weeks after surgery and he had a good prognosis and is presently on follow-up.

Jae-Young Yang et al: Sequential treatment from mandibulectomy to reconstruction on mandibular oral cancer - Case review II: mandibular anterior and the floor of the mouth lesion of basaloid squamous cell carcinoma and clear cell odontogenic carcinoma. J Korean Assoc Oral Maxillofac Surg 2021 

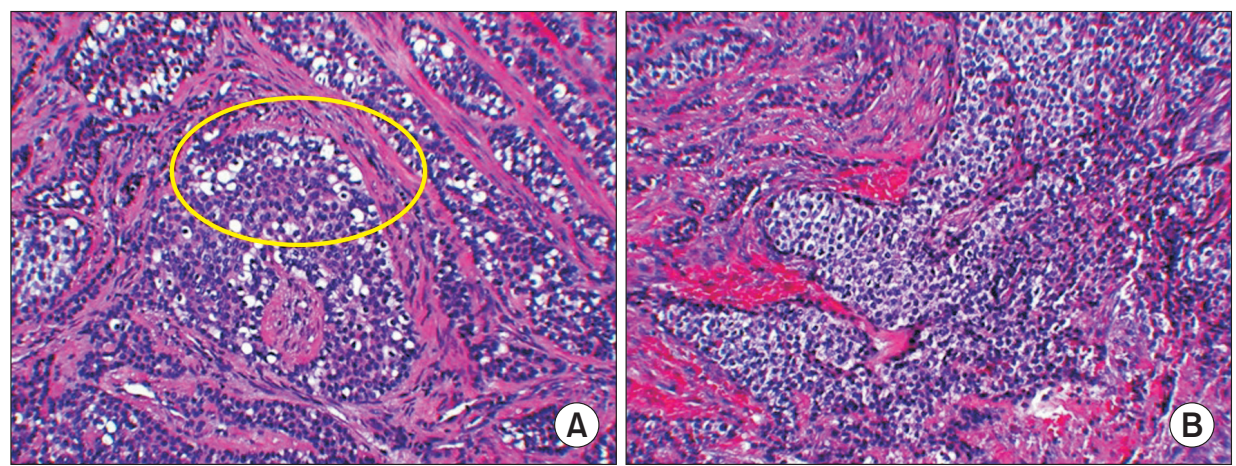

Fig. 6. Histopathologic findings (H\&E staining, $\times 200)$. A. The ameloblastic basal lamina structure was observed during the presence of clear cells. B. Hyperchromatic islands of basaloid epithelial cell have been demonstrated.

Jae-Young Yang et al: Sequential treatment from mandibulectomy to reconstruction on mandibular oral cancer-Case review II: mandibular anterior and the floor of the mouth lesion of basaloid squamous cell carcinoma and clear cell odontogenic carcinoma. $J$ Korean Assoc Oral Maxillofac Surg 2021
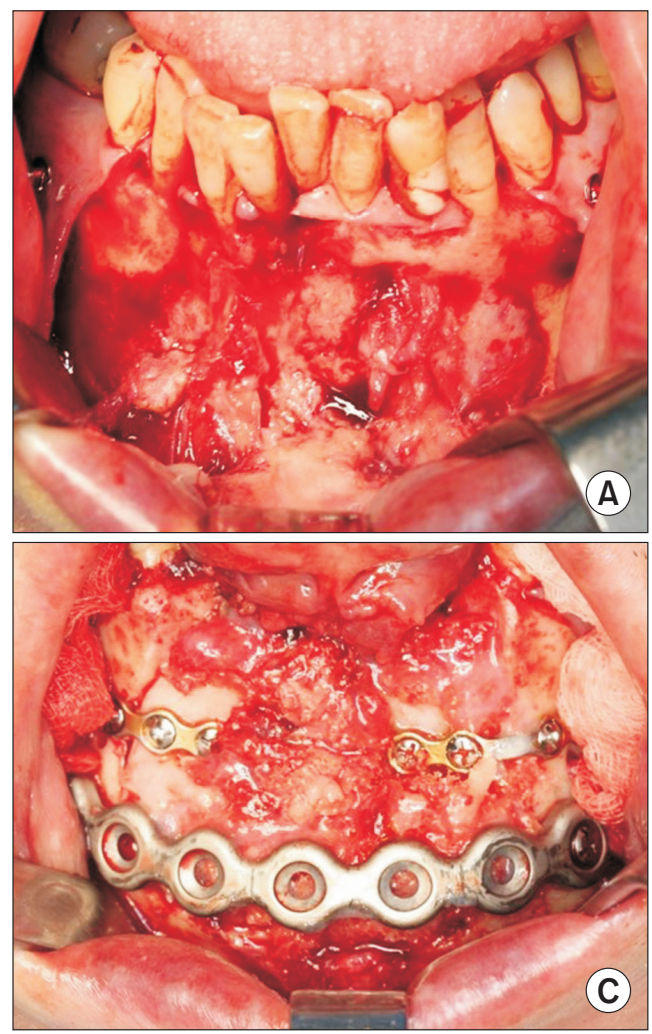
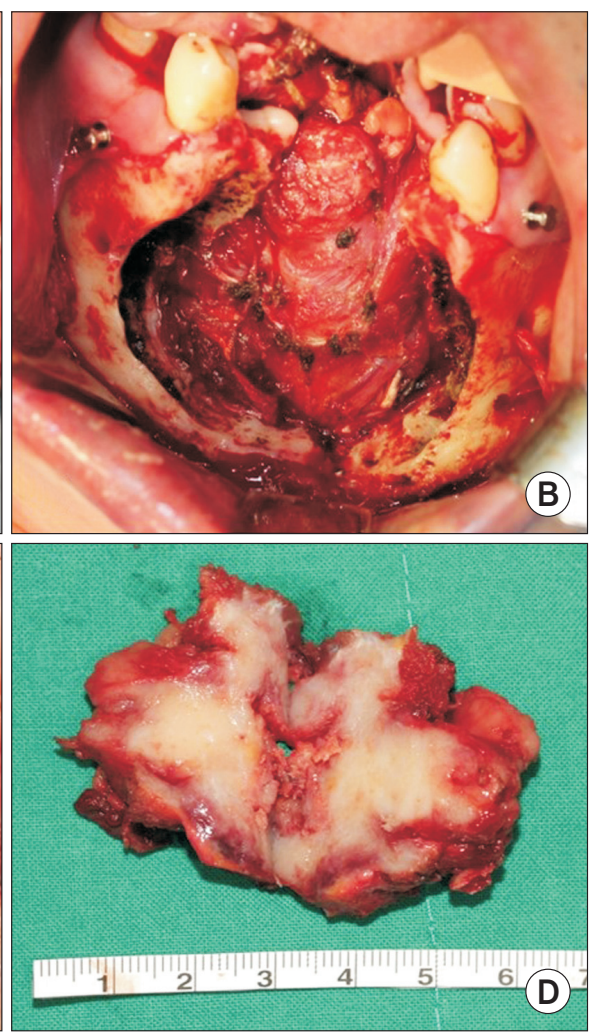

Fig. 7. Photographs of 1 st operation. A. Primary site before excision. B. After excision. C. Mandibular reconstruction with immediate iliac block bone graft and reconstruction plate. D. Main mass wide excision and mandibular reconstruction with iliac block bone were performed under general anesthesia.

Jae-Young Yang et al: Sequential treatment from mandibulectomy to reconstruction on mandibular oral cancer-Case review II: mandibular anterior and the floor of the mouth lesion of basaloid squamous cell carcinoma and clear cell odontogenic carcinoma. $J$ Korean Assoc Oral Maxillofac Surg 2021
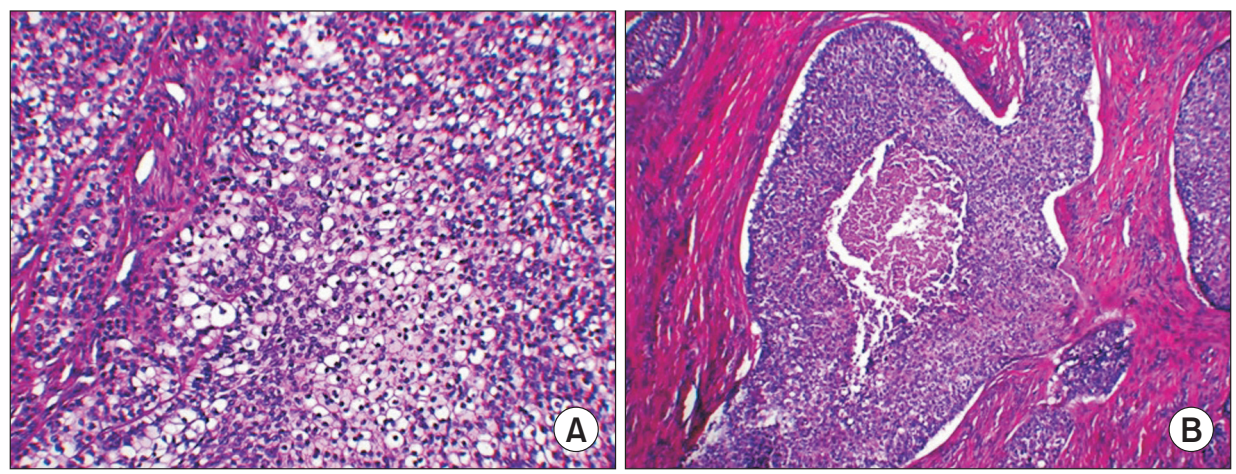

Fig. 8. Histopathologic findings of main mass. A. Abnormal differentiation of cells and cell island patterns was observed. Multiple clear cells showed dynamic mitosis as well as hyperchromatism, which made it possible to diagnose malignant tumors, clear cell odontogenic carcinoma (H\&E staining, $\times 200$ ). B. Histological finding of malignant tumor (H\&E staining, $\times 100$ ).

Jae-Young Yang et al: Sequential treatment from mandibulectomy to reconstruction on mandibular oral cancer - Case review II: mandibular anterior and the floor of the mouth lesion of basaloid squamous cell carcinoma and clear cell odontogenic carcinoma. J Korean Assoc Oral Maxillofac Surg 2021 

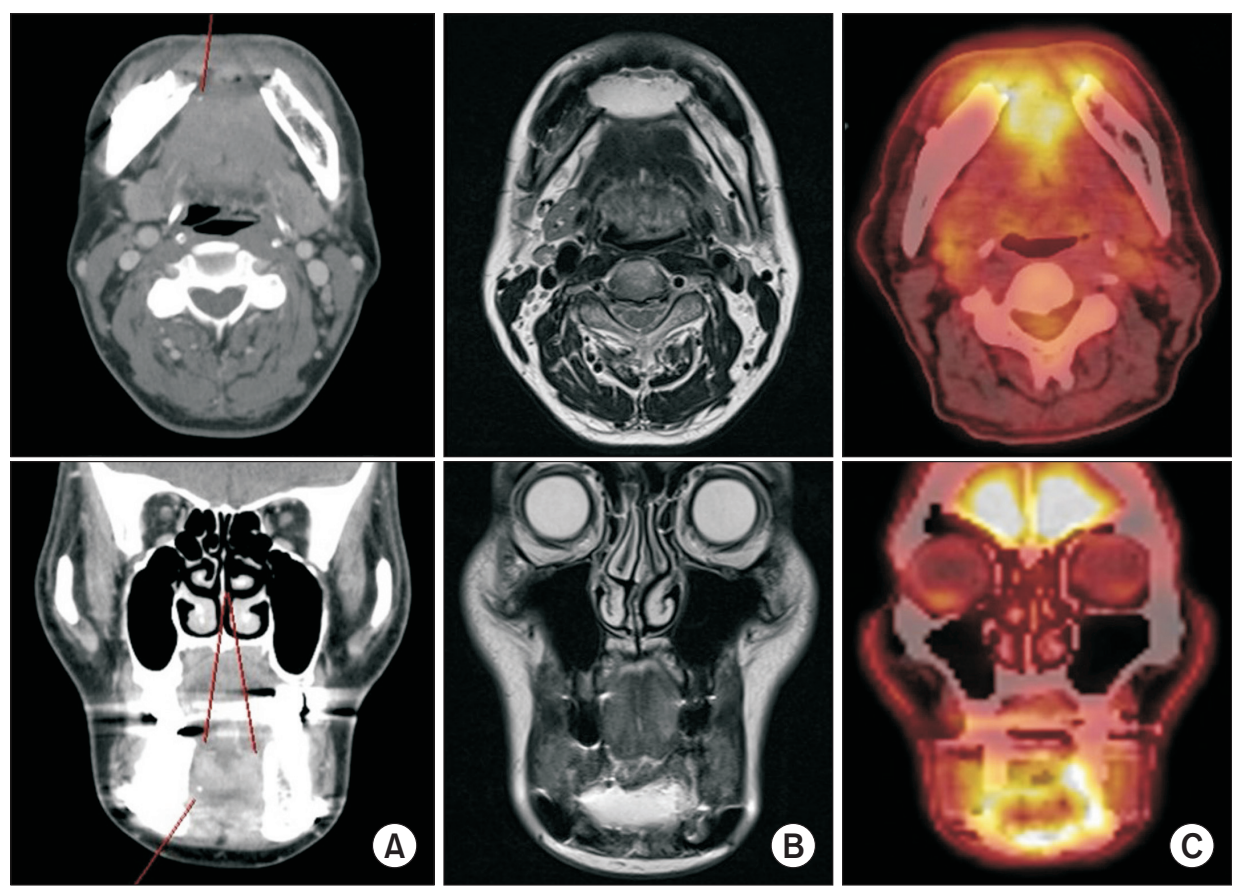

C

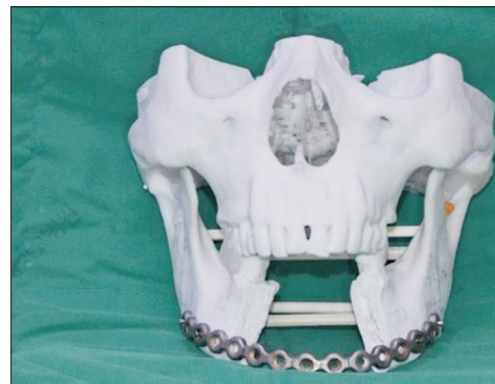

(A)
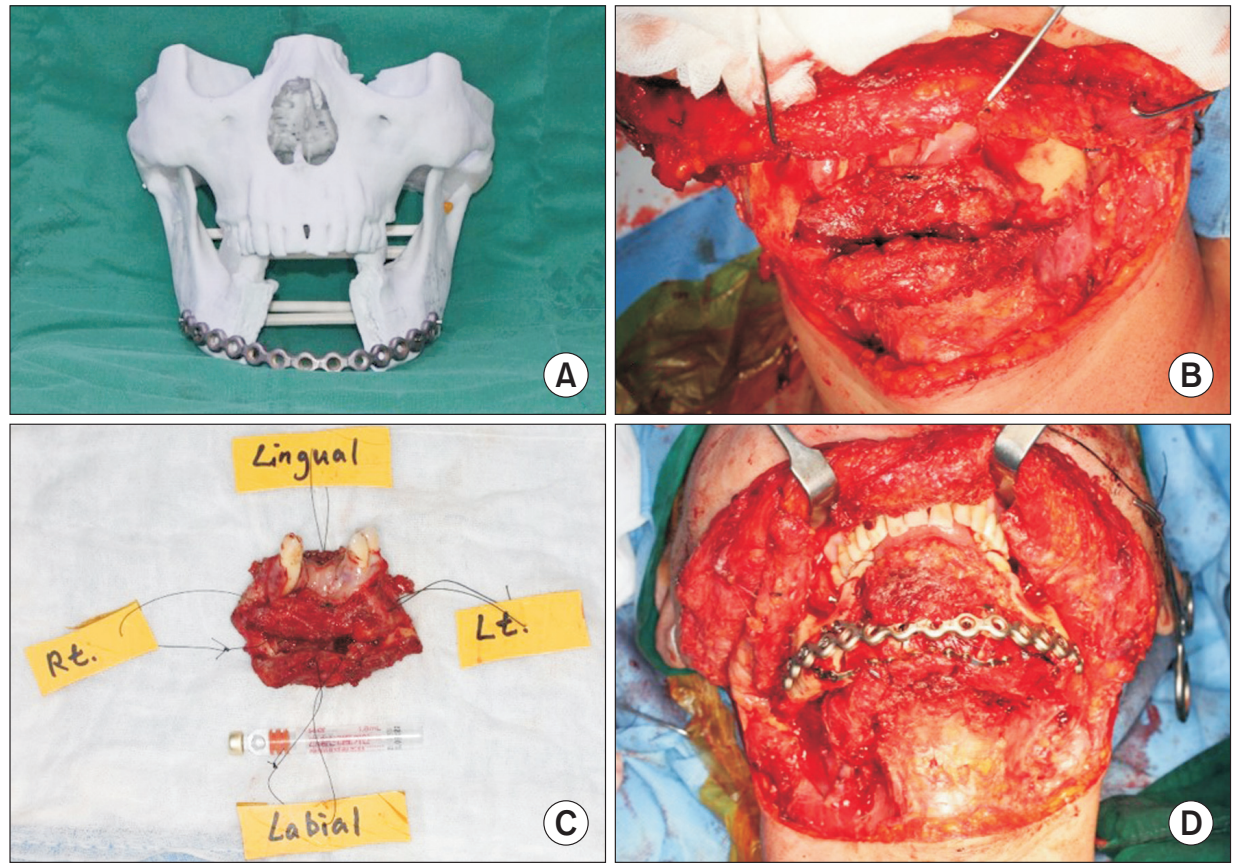

Fig. 9. Radiographs after 1st operation. A. Computed tomography (face) image. III-defined enhancing lesion at superior aspect of mandibular anterior, possibly involving both genioglossus muscles. B. Facial magnetic resonance image showed ill-defined enhancement at both genioglossus, hyoglossus muscle and diffuse swelling of the anteior aspect of mandibulectomy site. C. Abnormally increased fluorodeoxyglucose uptake was observed in mandibulectomy site. Jae-Young Yang et al: Sequential treatment from mandibulectomy to reconstruction on mandibular oral cancer - Case review II: mandibular anterior and the floor of the mouth lesion of basaloid squamous cell carcinoma and clear cell odontogenic carcinoma. $J$ Korean Assoc Oral Maxillofac Surg 2021
Fig. 10. Surgical procedure of 2 nd operation. A. Rapid prototype model of mandible. B. Segmental mandibulectomy with both supraomohyoid neck dissection (SOHND). C. Main mass. D. Mandibular reconstruction with fibula free flap with reconstruction plate and mini plates. Performed with segmental mandibulectomy with both SOHND included Levels I-III and mandible reconstruction with left fibula free flap.

Jae-Young Yang et al: Sequential treatment from mandibulectomy to reconstruction on mandibular oral cancer-Case review II: mandibular anterior and the floor of the mouth lesion of basaloid squamous cell carcinoma and clear cell odontogenic carcinoma. $J$ Korean Assoc Oral Maxillofac Surg 2021 of all malignant lesions. However, oral cavity BSCC is rare and can be indistinguishable from peripheral ameloblastoma due to the histopathological similarity between these disease entities $^{3,4}$. The most common treatment approach involves close follow-up for several years after wide excision. Oral cavity BSCC can show local recurrence and enlargement after conservative resection, as reported by Koutlas et al. ${ }^{5}$.

In contrast to the treatment available for BSCC (which is based on the current therapeutic principles for SCCs), pre- vious studies have proposed various protocols for CCOC. Conservative resection techniques, such as enucleation and curettage, have been used until CCOC was reclassified as a malignant tumor. After this categorization as a malignancy, many surgeons began to perform wide excision in these cases. Currently, LN involvement is common (as observed in our patient); therefore, neck dissection and surgical resection with a safety margin are the standard treatment approach used for $\mathrm{CCOC}^{6,7}$. 


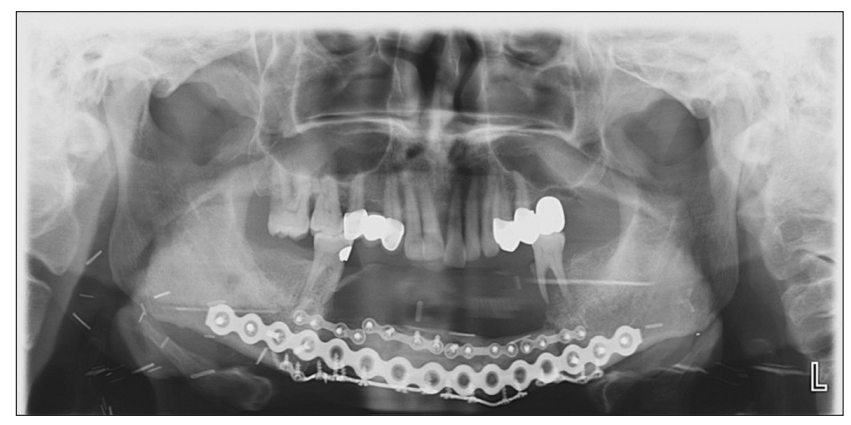

Fig. 11. Panoramic radiograph of after surgery. No recurrence was observed during reconstruction in favor of the mandible. Jae-Young Yang et al: Sequential treatment from mandibulectomy to reconstruction on mandibular oral cancer-Case review II: mandibular anterior and the floor of the mouth lesion of basaloid squamous cell carcinoma and clear cell odontogenic carcinoma. $J$ Korean Assoc Oral Maxillofac Surg 2021

Complete resection is essential for surgical treatment of any cancer. It is necessary to confirm the size and depth of the tumor and to establish the resection range by choosing a positive margin measuring 1 to $1.5 \mathrm{~cm}$ after confirmation of the diagnosis on $\mathrm{CT}$ and MRI. Prior to determining the extent of resection, the adjacent anatomical structures must be evaluated; careful preoperative decision-making is important to ensure preservation of these structures.

A radial forearm free flap or an anterolateral thigh flap can be useful in reconstruction of the tongue and floor of the mouth after resection without involvement of the adjacent bone. Accurate surgical treatment planning requires careful evaluation of potential mandibular invasion by primary tumors of the oral cavity. The mandible is at risk of invasion in patients with primary tumors overlying and adherent to the mandible. Advanced tumors of the mandible require marginal mandibulectomy or segmental mandibulectomy after confirmation using bone scans and CT. Reconstruction using a fibula free flap can be considered to restore the continuity of the mandible following segmental mandibulectomy ${ }^{8}$.

Successful mandibular reconstruction is necessary to ensure satisfactory postoperative cosmetic outcomes and to restore optimal function, including speech and mastication; therefore, this surgery is a complicated and challenging procedure, ${ }^{9,10}$. In this study, we performed immediate reconstruction using a fibula free flap and a reconstruction plate. Currently, it is possible to fabricate the fibula and reconstruction plate using computer-aided design and computer-aided technology. However, in our case, the preoperative rapid prototype (RP) model was used as a reconstructive plate after cutting the plate to match the size of the bone defect.

The associated easy application, low risk, short surgical time, stability, aesthetic satisfaction, and low costs postopera- tively are some advantages of using pre-bent titanium plates in reconstruction. However, non-union, instability, and infection are known complications in such cases ${ }^{11}$.

This study highlights the role of simulated surgery using an RP model and a referenceable fibular resin model. A free flap was designed using measurements that were applied to the fabricated fibula resin model. The RP model combined with a fibula resin model for simulation was useful to save time and could reduce perioperative errors. Moreover, we successfully used a pre-bent reconstruction plate and a guided fibula resin model to allow immediate reconstruction during conventional surgery ${ }^{12}$.

\section{Conclusion}

Treatment options should be considered for malignant tumors of the anterior mandible (including those of the floor of the mouth) depending on the extent of bone involvement and neck node involvement: (a) segmental mandibulectomy or marginal mandibulectomy for resection of mandibular lesions using the fibula free flap or anterolateral thigh free flap method for surgical defect reconstruction and (b) routine neck node dissection to treat tumors of the floor of the mouth and the anterior mandible and to remove occult metastatic nodes. For simultaneous anterior mandibular and floor of the mouth reconstruction using a fibula free flap, recipient vessels (including the superior thyroid and the external jugular veins) must be selected after neck dissection on the side of the neck contralateral to the leg from which the fibula is harvested. For example, recipient vessels of the right side of the neck should be matched to the peroneal donor vessels of the left fibula free flap due to reverse up-down repositioning of the skin pedicle on the left fibular flap to cover the soft tissue defect of the floor of the mouth and a right positional change of the end of the donor peroneal vessels on the left fibular flap to allow for anastomosis of the right neck vessels.

Mandibular reconstruction using a fibula free flap in patients with anterior mandibular and floor of the mouth cancer can achieve good functional and cosmetic outcomes. Attention to each step of the oral flap transfer procedure is important for achieving successful results; however, reconstruction plate pre-plating and RP model templating should be considered crucial steps that facilitate the procedure and foster satisfactory outcomes of reconstruction. 


\section{ORCID}

Jae-Young Yang, https://orcid.org/0000-0002-8057-7605

Dae-Seok Hwang, https://orcid.org/0000-0001-6899-1769

Uk-Kyu Kim, https://orcid.org/0000-0003-1251-7843

\section{Authors' Contributions}

J.Y.Y. participated in data collection and wrote the manuscript. D.S.H. contributed to conception and revision of the manuscript. U.K.K. provided with clinical data, participated in the study design and data analysis. All authors read and approved the final manuscript.

\section{Acknowledgements}

This study was supported by National Research Foundation of Korea and funded by the Korea government (No. 2019R1F1A1042528).

\section{Ethics Approval and Consent to Participate}

The study was approved by the institutional review board of Pusan National University Dental Hospital (IRB No. PNUDH-2020-034). Informed consent was waived by the IRB.

\section{Consent for Publishing Photographs}

Written informed consent was obtained from the patients for publication of this article and accompanying images.

\section{Conflict of Interest}

No potential conflict of interest relevant to this article was reported.

\section{References}

1. Candia J, Fernández A, Kraemer K. Survival and mortality from oral cancer by anatomical location. A narrative review. J Oral Res 2016;5:35-42. https://doi.org/10.17126/joralres.2016.007

2. Sankaranarayanan R. Oral cancer in India: an epidemiologic and clinical review. Oral Surg Oral Med Oral Pathol 1990;69:325-30. https://doi.org/10.1016/0030-4220(90)90294-3

3. Wu S, Han J, Li WQ, Li T, Qureshi AA. Basal-cell carcinoma incidence and associated risk factors in U.S. women and men. Am J Epidemiol 2013;178:890-7. https://doi.org/10.1093/aje/kwt073

4. Woods TR, Cohen DM, Islam MN, Kratochvil FJ, Stewart JC, Reeder SL, et al. Intraoral basal cell carcinoma, a rare neoplasm: report of three new cases with literature review. Head Neck Pathol 2014;8:339-48. https://doi.org/10.1007/s12105-013-0505-5

5. Koutlas IG, Koch CA, Vickers RA, Brouwers FM, Vortmeyer AO. An unusual ostensible example of intraoral basal cell carcinoma. J Cutan Pathol 2009;36:464-70. https://doi.org/10.1111/j.16000560.2008.01059.x

6. Kim M, Cho E, Kim JY, Kim HS, Nam W. Clear cell odontogenic carcinoma mimicking a cystic lesion: a case of misdiagnosis. J Korean Assoc Oral Maxillofac Surg 2014;40:199-203. https://doi. org/10.5125/jkaoms.2014.40.4.199

7. Ebert CS Jr, Dubin MG, Hart CF, Chalian AA, Shockley WW. Clear cell odontogenic carcinoma: a comprehensive analysis of treatment strategies. Head Neck 2005;27:536-42. https://doi. org/10.1002/hed.20181

8. Shah JP, Gil Z. Current concepts in management of oral cancer-surgery. Oral Oncol 2009;45:394-401. https://doi.org/10.1016/ j.oraloncology.2008.05.017

9. Erdmann D, Giessler GA, Bergquist GE, Bruno W, Young H, Heitmann C, et al. [Free fibula transfer. Analysis of 76 consecutive microsurgical procedures and review of the literature]. Chirurg 2004;75:799-809. German. https://doi.org/10.1007/s00104-004$0833-9$

10. Wallace CG, Chang YM, Tsai CY, Wei FC. Harnessing the potential of the free fibula osteoseptocutaneous flap in mandible reconstruction. Plast Reconstr Surg 2010;125:305-14. https://doi. org/10.1097/PRS.0b013e3181c2bb9d

11. Harjani B, Singh RK, Pal US, Singh G. Locking v/s non-locking reconstruction plates in mandibular reconstruction. Natl J Maxillofac Surg 2012;3:159-65. https://doi.org/10.4103/0975-5950.111371

12. Park HK, Kim TS, Geum DH, Yoon SY, Song JM, Hwang DS, et al. Mandibular intraosseous squamous cell carcinoma lesion associated with odontogenic keratocyst: a case report. J Korean Assoc Oral Maxillofac Surg 2015;41:78-83. https://doi.org/10.5125/ jkaoms.2015.41.2.78 